\title{
HPV-Impfung für Jungen: Streit um Kosteneffizienz
}

\begin{abstract}
Seit es einen Impfstoff gegen verschiedene Typen von humanen Papillomviren (HPV) gibt, gibt es auch Diskussionen, ob neben Mädchen und jungen Frauen auch Knaben und junge Männer geimpft werden sollten. Über das Für und Wider einer solchen Impfung ist auf der 2. CoVAC-Konferenz in München gestritten worden.
\end{abstract}

— Sollte man junge Männer gegen HPV impfen? Zu dieser Frage existiert derzeit kein Konsens der Fachleute. In Deutschland wird eine HPV-Schutzimpfung zwar für Mädchen, aber nicht für Jungen angeboten. In den Nachbarländern Österreich und Schweiz überlegen die zuständigen Gremien jedoch intensiv, die Impfung auf Jungen auszuweiten, da schließlich auch Männer Träger und Überträger von HPV sind.

\section{Was dafür spricht ...}

Daran, dass die Impfung bei Männern wirkt, besteht nach den vorliegenden Studien kein Zweifel. Auf der Konferenz in München fasste Prof. Xavier Bosch, Barcelona, die Argumente zusammen, die in seinen Augen für eine HPV-Impfung von Jungen sprechen. Zum einen ist da die hohe Prävalenz von HPV-Infektionen unter Männern mit bis zu $70 \%$. Im Gegensatz zu Frauen, wo die Prävalenz mit dem Alter sinkt, bleibt diese Rate bei Männern über die Jahre stabil.

Bosch plädierte dafür, beim Krebsrisiko durch HPV nicht nur das Zervixkarzinom in den Blick zu nehmen. Eine Beteiligung von HPV gebe es schließlich auch beim Analkrebs (84,3\%), Peniskarzinom (47\%), bei Oropharyngeal- $(35,6 \%)$ und Mundhöhlenkrebs (23,5\%). Der katalanische Onkologe wies darauf hin, dass oropharyngeale Karzinome im Jahr 2010 zum Zervixkrebs aufgeschlossen haben, was die Krankheitshäufigkeit betrifft. Beide Tumorarten standen damals mit einer Quote von 10/100000 zu Buche. Seither hat die Erkrankungsrate von Oropharyngealkrebs weiter zugenommen. In 70-90\% aller Krebsfälle, bei denen HPV involviert ist, sind die Typen 16 und 18 nachweisbar. Was also sollte gegen eine Impfung von Jungen sprechen?

\section{... und was dagegen}

Gegen die Impfung sprechen die Kosten. Das unterstrich Yvonne Deleré vom Berliner Robert Koch-Institut. Sie rechnete vor, dass die Kosten pro qualitätskorrigiertem Lebensjahr von 15000 auf 120000 Euro steigen würden, wenn nicht nur 50\% der Mädchen, sondern auch $50 \%$ der Jungen geimpft würden. Es sei eben ein Unterschied, ob man bei einer Maßnahme wie der HPV-Impfung das Individuum oder die gesamte Population betrachte. Soll heißen: Effektiv ist nicht unbedingt auch effizient.

Die Infektionsepidemiologin untermauerte ihre Skepsis mit Zahlen. Hiernach ist etwa bei Condylomata accuminata mit einer jährlichen Erkrankungsrate von 150/100000 $\mathrm{zu}$ rechnen. Die HPV-Impfung hat hier tatsächlich einen Wirkungsgrad von 89,4\%, „Doch die Erkrankung ist benigne", meinte Deleré. Die Quote für Analkrebs liegt bei 1-2/100 000. Die Effektivität der Impfung erreicht hier $54,9 \%$. Laut Deleré bezieht sich diese Zahl aber auf anale intraepitheliale Neoplasien von schwulen Männern, nicht auf die männliche Allgemeinpopulation. Für andere HPV-assoziierte Krebsformen lägen nur Schätzungen zur Effektivität der Impfung vor.

\section{Besser die Impfraten} der Mädchen erhöhen

Aus all dem schloss die RKI-Expertin: „Die Durchimpfungsrate der Mädchen zu erhöhen, scheint sinnvoller zu sein, als Jungen zu impfen." Sind nämlich genügend Frauen geimpft, kommt es zu

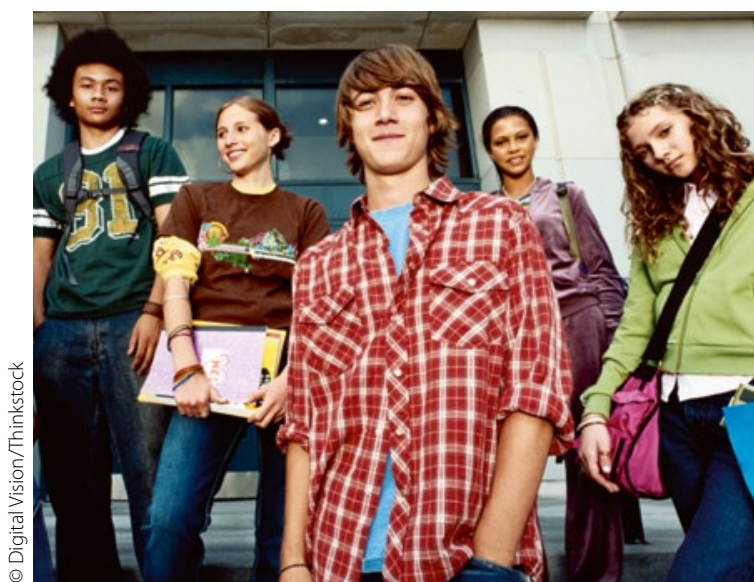

Bei Jungen nur auf den Herdenschutz vertrauen?

einem Herdeneffekt. Er schützt Männer auch dann, wenn sie selbst nicht geimpft sind. 2007 startete in Australien ein HPV-Impfprogramm für Mädchen und Frauen im Alter von 12 bis 26 Jahren. Das hatte Konsequenzen für die Männer: Die Zahl heterosexueller Männer, bei denen Genitalwarzen diagnostiziert wurden, sank in der Folgezeit um 28\% [Donovan B et al. Lancet Infect Dis 2011; 11: 39-44]. Die Impfrate der Frauen hatte bei $65,1 \%$ gelegen.

Deleré hält es für unwahrscheinlich, dass hierzulande ein HPV-Impfprogramm für Jungen eingeführt wird. Die mathematischen Modelle zur Kosteneffizienz gehen allerdings von einer Durchimpfungsrate von etwa $80 \%$ bei den Frauen aus. Davon ist Deutschland weit entfernt: Die aktuellen Impfraten liegen unter 50\%. Für Deleré ist das dennoch kein Grund, die Impfung von Jungen ins Spiel zu bringen, im Gegenteil. Sie fragte: „Wieso sollten wir Jungen ein Impfprogramm anbieten, das schon bei Mädchen nicht recht funktioniert?"

DR. ROBERT BUBLAK -

\footnotetext{
- The 2nd Conference on Controversies in Vaccination in Adults, München, 14.-17. 2. 2013
} 